\title{
Propagation of Axisymmetric Stoneley Waves in Elastic Solids
}

\author{
Chunlei Bian $^{a}$, Bin Huang ${ }^{a, b, c}$, Longtao XiE ${ }^{a, b, c}$, \\ LIJUN YI ${ }^{a, b, c}$, LILI YUAN ${ }^{a, d}$ AND JI WANG ${ }^{a, b, c, *}$ \\ ${ }^{a}$ Piezoelectric Device Laboratory, School of Mechanical Engineering and Mechanics, \\ Ningbo University, 818 Fenghua Road, Ningbo, 315211 Zhejiang, China \\ ${ }^{b}$ TXC-NBU Joint Center of Research, School of Mechanical Engineering and Mechanics, \\ Ningbo University, 818 Fenghua Road, Ningbo, 315211 Zhejiang, China \\ ${ }^{c}$ Key Laboratory of Impact and Safety Engineering of Ministry of Education, Ningbo University, \\ 818 Fenghua Road, Ningbo, 315211 Zhejiang, China \\ ${ }^{d}$ School of Civil and Environmental Engineering, Ningbo University, 818 Fenghua Road, Ningbo, \\ 315211 Zhejiang, China
}

Received: 10.10.2020 \& Accepted: 07.12.2020

Doi: 10.12693/APhysPolA.139.124

*e-mail: wangji@nbu.edu.cn

\begin{abstract}
Stoneley waves - as one of just a few special wave modes propagating in infinite elastic solids and interfaces - are well-known for their existence and frequency with distinct features. Their analysis and properties are usually presented through the formulation in Cartesian coordinates, while the essential features including the phase velocity and wave patterns are also consistent with other coordinates based on an equivalent principle. The variation of the Stoneley wave properties with different coordinate framework should be examined for possible insights related to mathematical solutions and applications in addition to known knowledge. Through a systematic formulation with cylindrical coordinates and subsequent solutions in the Bessel functions, it is shown that the amplitudes of Stoneley waves in an axisymmetric configuration will decrease with radius which is in a strong contrast to the Cartesian coordinate case. The examination of such features in a systematic manner can be of importance in crafting and tuning of engineering applications.
\end{abstract}

topics: Stoneley wave, propagation, solid, velocity

\section{Introduction}

Through rigorous studies of wave propagation in earlier work by pioneers, a few typical wave modes with distinct features and solutions have been known as part of the theory and applications of the wave phenomenon in elastic solids of different configurations. Among few widely known wave modes, the Stoneley waves are well presented and examined in scientific literature for their unique features like the existence with interfaces of solids and fluids and the fact that they are nondispersive with material properties.

In the case of the wave patterns and properties of elastic solids and structures different from rectangular shapes, it is necessary to study the wave propagation with an appropriate choice of coordinate systems, which was described in popular textbooks and monographs by, e.g., Ewing [1], Achenbach [2], Graff [3], Eringen [4] and Rose [5], and in dissertations [6-9] with details on some specific wave modes. Surprisingly, details of axisymmetric waves are not referred to in the major literature (including the papers mentioned above) as the essential modes we are thoroughly familiar with [10]. However, it does not mean that there are difficulties in obtaining solutions and making necessary comparisons with known results in Cartesian coordinates. This reflects a general conviction that the solutions and their essential features are well known and the same. This, of course, is correct to some degree but the coordinate system dependence has specific features which can easily be hidden if not examined in detail as shown in [11]. It is found that in the case of axisymmetric Rayleigh waves, the trajectory of particles is no longer elliptical and the deformation will also decrease along the radius. This is quite different from the solutions in a Cartesian coordinate system but it also reveals that wave patterns are the same for a larger radius. Clearly, it shows that there are some special features in association with the Bessel functions to be better understood through comparison with a different coordinate framework. With this objective in mind, this study focuses on Stoneley waves at the interface of two semi-infinite elastic solids with cylindrical coordinates for axisymmetric solutions and features. 


\section{Governing equations and solutions with cylindrical coordinates}

Stoneley waves exist at the interface of two perfectly bounded semi-infinite elastic solids made of different materials, satisfying the given conditions [12-15]. As a result, there exist a unique velocity, a distinct wave mode and wave modes in both materials at the interface. We now consider the axisymmetric waves which propagate along the radius and their decay along the depth using cylindrical coordinates. The two elastic infinite-half-spaces are bonded together along the plane $z=0$ [5], as sketched in Fig. 1. The quantities $\bar{\rho}$ and $\rho$ denote the mass density corresponding to the up and down half-space. Notably, also $\bar{\lambda}, \bar{\mu}$ and $\lambda, \mu$ can be responsible for the elastic properties, i.e., the Lamé constants.

Owing to the axisymmetric property, for this problem the angular coordinate $\theta$ and the circumferential displacement component $u_{\theta}$ are neglected in the analysis. As a result, the displacement vector can be simplified to

$$
\boldsymbol{u}(r, z, t)=u_{r}(r, z, t) \boldsymbol{e}_{r}+u_{z}(r, z, t) \boldsymbol{e}_{z}
$$

with $u_{\theta}=0$. The displacements $u_{r}, u_{\theta}$ and $u_{z}$ read as the function of the respective coordinate $r, \theta, z$, while $t$ is the time.

Using the Helmholtz decomposition, we introduce a scalar potential $\phi$ and a vector potential $\boldsymbol{H}$, where $\boldsymbol{H}=H_{r} \boldsymbol{e}_{r}+H_{\theta} \boldsymbol{e}_{\theta}+H_{z} \boldsymbol{e}_{z}$ to simplify the problem for possible solutions [1]. With (1), we only need to retain the component $H_{\theta}$ in $\boldsymbol{H}$, so the displacements can be decomposed into [16]:

$$
\boldsymbol{u}(r, z, t)=\nabla \phi(r, z, t)+\nabla \times\left(H_{\theta}(r, z, t) \boldsymbol{e}_{\theta}\right)
$$

where

$$
\begin{aligned}
& \nabla \phi=\frac{\partial \phi}{\partial r} \boldsymbol{e}_{r}+\frac{\partial \phi}{\partial z} \boldsymbol{e}_{z} \\
& \nabla \times\left(H_{\theta} \boldsymbol{e}_{\theta}\right)=-\frac{\partial H_{z}}{\partial r} \boldsymbol{e}_{r}+\frac{\partial \phi}{\partial z} \boldsymbol{e}_{z} .
\end{aligned}
$$

To clear up,

$$
\begin{gathered}
\boldsymbol{u}(r, z, t)=\left(\frac{\partial \phi}{\partial r}-\frac{\partial H_{\theta}}{\partial z}\right) \boldsymbol{e}_{r} \\
+\left(\frac{\partial \phi}{\partial z}+\frac{1}{r} \frac{\partial\left(r H_{\theta}\right)}{\partial r}\right) \boldsymbol{e}_{z} .
\end{gathered}
$$

Now, by substituting this displacement vector $\boldsymbol{u}$ in the Lamé equations of motion of elasticity $[17,18]$ :

$$
(\lambda+\mu) \nabla(\nabla \boldsymbol{u})+\mu \nabla^{2} \boldsymbol{u}=\rho \ddot{\boldsymbol{u}},
$$

the problem is simplified to two governing equations [14]:

$$
\begin{aligned}
& \nabla^{2} \phi=\frac{1}{c_{L}^{2}} \ddot{\phi}, \\
& \nabla^{2} H_{\theta}-\frac{H_{\theta}}{r^{2}}=\frac{1}{c_{T}^{2}} \ddot{H}_{\theta},
\end{aligned}
$$

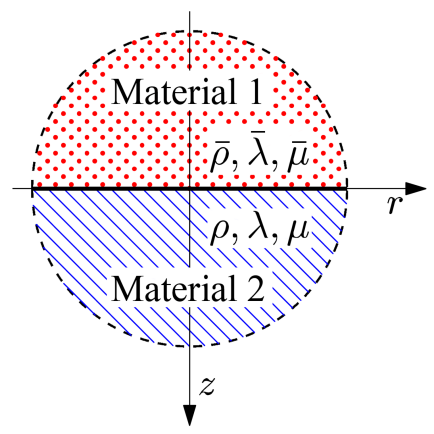

Fig. 1. The interface of two perfectly bounded semi-infinite elastic solids in cylindrical coordinates.

where

$$
c_{L}=\sqrt{\frac{\lambda+2 \mu}{\rho}} \text { and } c_{T}=\sqrt{\frac{\mu}{\rho}}
$$

are the longitudinal and transverse wave velocities, respectively.

In the meantime, the same description in the cylindrical coordinate system is as follows:

$$
\begin{aligned}
& \nabla^{2} \phi=\frac{1}{r} \frac{\partial}{\partial r}(r \phi)+\frac{\partial^{2} \phi}{\partial z^{2}}, \\
& \nabla^{2} H_{\theta}=\frac{1}{r} \frac{\partial}{\partial r}\left(r H_{\theta}\right)+\frac{\partial^{2} H_{\theta}}{\partial z^{2}} .
\end{aligned}
$$

Let us further introduce the transformation

$$
H_{\theta}=-\frac{\partial \psi(r, z, t)}{\partial r}
$$

with the use of which

$$
\begin{gathered}
\boldsymbol{u}(r, z, t)=\left(\frac{\partial \phi}{\partial r}+\frac{\partial^{2} \psi}{\partial r^{2}}\right) \boldsymbol{e}_{r} \\
+\left(\frac{\partial \phi}{\partial z}+\frac{\partial^{2} \psi}{\partial z^{2}}-\frac{1}{c_{T}^{2}} \ddot{\psi}\right) \boldsymbol{e}_{z} .
\end{gathered}
$$

Hence, the equivalence of (8) becomes simply

$$
\nabla^{2} \psi=\frac{\ddot{\psi}}{c_{T}^{2}}+g(z)
$$

where $g(z)$ is an arbitrary function of $z$. Assuming $g(z)=0$, one arrives at

$$
\nabla^{2} \psi=\frac{\ddot{\psi}}{c_{T}^{2}} .
$$

Summing up this part, the equivalent form of (7) and (8) is then

$$
\begin{aligned}
& \nabla^{2} \phi=\frac{1}{c_{L}^{2}} \ddot{\phi}, \\
& \nabla^{2} \psi=\frac{1}{c_{T}^{2}} \ddot{\psi} .
\end{aligned}
$$

The above equations are the standard wave propagation equations with wave velocities $c_{L}$ and $c_{T}$. Since the two equations, (16) and (17), are the same, we need to solve the first equation only. Employing the method of separation of variables through

$$
\phi=\phi(r, z, t)=R(r) Z(z) \mathrm{e}^{\mathrm{i} \omega t},
$$


where $\omega$ is the angular frequency of waves, this brings us to

$$
\begin{aligned}
& \frac{\omega^{2}}{c_{L}^{2}} R(r) Z(z)+Z(z) \frac{\mathrm{d}^{2} R(r)}{\mathrm{d} r^{2}} \\
& \quad+\frac{Z(z)}{r} \frac{\partial R(r)}{\partial r}+R(r) \frac{\mathrm{d}^{2} Z(z)}{\mathrm{d} z^{2}}=0 .
\end{aligned}
$$

Here, the following definition of the wave number is used:

$$
k=\frac{\omega}{c} .
$$

Then, the potential function can be given as

$$
\phi(r, z, t)=\left(A_{1} \mathrm{e}^{\alpha z}+A_{2} \mathrm{e}^{-\alpha z}\right) J_{0}(k r) \mathrm{e}^{\mathrm{i} \omega t},
$$

where $A_{1}$ and $A_{2}$ are the undetermined constants, $\alpha$ is the decaying constant satisfying the relation $k^{2}=\alpha^{2}+\omega^{2} / c_{L}^{2}$ and $J_{0}(k r)$ is the zeroth-order Bessel function of the first kind.

Similarly, we have

$$
\psi(r, z, t)=\left(B_{1} \mathrm{e}^{\beta z}+B_{2} \mathrm{e}^{-\beta z}\right) J_{0}(k r) \mathrm{e}^{\mathrm{i} \omega t},
$$

where $B_{1}$ and $B_{2}$ are the undetermined constants and $\beta$ is the decaying constant satisfying the relation $k^{2}=\beta^{2}+\omega^{2} / c_{T}^{2}$.

Obviously, the exponential function $\mathrm{e}^{\alpha z}$ should be neglected in the postulate function form because of unbounded solution at $z \rightarrow \infty$. Thus,

$$
\begin{aligned}
& \phi(r, z, t)=A \mathrm{e}^{-\alpha z} J_{0}(k r) \mathrm{e}^{\mathrm{i} \omega t}, \\
& \psi(r, z, t)=B \mathrm{e}^{-\beta z} J_{0}(k r) \mathrm{e}^{\mathrm{i} \omega t} .
\end{aligned}
$$

A substitution of (23) and (24) into the displacement functions (5) allows to determine the displacement fields, i.e.,

$$
\begin{gathered}
u_{r}=\frac{\partial \phi}{\partial r}+\frac{\partial^{2} \psi}{\partial r^{2}}= \\
-k\left(A \mathrm{e}^{-\alpha z}-\beta B \mathrm{e}^{-\beta z}\right) J_{1}(k r) \mathrm{e}^{\mathrm{i} \omega t}, \\
u_{z}=\frac{\partial \phi}{\partial z}+\frac{\partial^{2} \psi}{\partial z^{2}}-\frac{\ddot{\psi}}{c_{S}^{2}}= \\
\quad\left(-\alpha A \mathrm{e}^{-\alpha z}+k^{2} B \mathrm{e}^{-\beta z}\right) J_{0}(k r) \mathrm{e}^{\mathrm{i} \omega t} . \\
\text { Here, } \quad k=k q, \quad \beta=k q
\end{gathered}
$$

and

$$
\begin{aligned}
& p=\sqrt{1-\left(\frac{c}{c_{L}}\right)^{2}}, \\
& q=\sqrt{1-\left(\frac{c}{c_{T}}\right)^{2}} .
\end{aligned}
$$

Consequently, the stress components are

$$
\begin{aligned}
& \sigma_{z}=\lambda \nabla \cdot \boldsymbol{u}+2 \mu \frac{\partial u_{z}}{\partial z}= \\
& \mu\left[\left(k^{2}+\beta^{2}\right) A \mathrm{e}^{-\alpha z}-2 k^{2} \beta B \mathrm{e}^{-\beta z}\right] J_{0}(k r) \mathrm{e}^{\mathrm{i} \omega t} \\
& \tau_{z r}=\mu\left(\frac{\partial u_{r}}{\partial z}+\frac{\partial u_{z}}{\partial r}\right)= \\
& k \mu\left[2 \alpha A \mathrm{e}^{-\alpha z}-\left(k^{2}+\beta^{2}\right) B \mathrm{e}^{-\beta z}\right] J_{1}(k r) \mathrm{e}^{\mathrm{i} \omega t}
\end{aligned}
$$


Cartesian coordinate solutions can also be compared with known results. The amplitude rations of (34) are

$$
\frac{\bar{A}}{\bar{B}}=-k \bar{n}, \quad \frac{A}{B}=-k n, \quad \frac{B}{\bar{B}}=h,
$$

where

$$
\begin{aligned}
& \bar{n}=\frac{2 p(q+\bar{q})-\left(1+q^{2}\right)(1+p \bar{q})+\bar{g}\left(1+\bar{q}^{2}\right)(1-p q)}{2 p(1+\bar{p} q)-\left(1+q^{2}\right)(p+\bar{p})+2 \bar{g} \bar{p}(1-p q)}, \\
& n=\frac{\bar{n}(1+\bar{p} q)-(q+\bar{q})}{\bar{n}(p+\bar{p})-(1+p \bar{q})}, \quad h=\frac{\bar{n}-\bar{q}}{n+q}
\end{aligned}
$$

Naturally, the displacement field and stress field are

$$
\begin{aligned}
& u_{r}=h k^{2} \bar{B}\left[n \mathrm{e}^{-\alpha z}+q \mathrm{e}^{-\beta z}\right] J_{1}(k r) \mathrm{e}^{\mathrm{i} \omega t} \\
& \bar{u}_{r}=k^{2} \bar{B}\left[\bar{n} \mathrm{e}^{\bar{\alpha} z}-\bar{q} \mathrm{e}^{\bar{\beta} z}\right] J_{1}(k r) \mathrm{e}^{\mathrm{i} \omega t}, \\
& u_{z}=h k^{2} \bar{B}\left[n p \mathrm{e}^{-\alpha z}+q \mathrm{e}^{-\beta z}\right] J_{0}(k r) \mathrm{e}^{\mathrm{i} \omega t}, \\
& \bar{u}_{z}=k^{2} \bar{B}\left[-\bar{n} \bar{p} \mathrm{e}^{\bar{\alpha} z}+\mathrm{e}^{\bar{\beta} z}\right] J_{0}(k r) \mathrm{e}^{\mathrm{i} \omega t},
\end{aligned}
$$

and

$$
\begin{aligned}
& \sigma_{z}=-\mu k^{3} h \bar{B}\left[\left(1+q^{2}\right) n \mathrm{e}^{-\alpha z}+2 q \mathrm{e}^{-\beta z}\right] \\
& \quad \times J_{0}(k r) \mathrm{e}^{\mathrm{i} \omega t}, \\
& \bar{\sigma}_{z}=\bar{\mu} k^{3} \bar{B}\left[-\left(1+\bar{q}^{2}\right) \bar{n} \mathrm{e}^{\bar{\alpha} z}+2 \bar{q} \mathrm{e}^{\bar{\beta} z}\right] \\
& \quad \times J_{0}(k r) \mathrm{e}^{\mathrm{i} \omega t}, \\
& \tau_{z r}=-\mu k^{3} h \bar{B}\left[2 n p \mathrm{e}^{-\alpha z}+\left(1+q^{2}\right) \mathrm{e}^{-\beta z}\right] \\
& \quad \times J_{1}(k r) \mathrm{e}^{\mathrm{i} \omega t}, \\
& \bar{\tau}_{z r}=\bar{\mu} k^{3} \bar{B}\left[2 \bar{n} \bar{p} \mathrm{e}^{\bar{\alpha} z}-\left(1+\bar{q}^{2}\right) \mathrm{e}^{\bar{\beta} z}\right] \\
& \quad \times J_{1}(k r) \mathrm{e}^{\mathrm{i} \omega t} .
\end{aligned}
$$

The complete solutions of Stoneley waves have been presented. Knowing the properties of two materials, one can systematically calculate the wave velocity along the displacement and stress fields. This, of course, could reveal the difference of Stoneley waves in the structure shown in Fig. 1.

\section{Numerical examples}

Now we consider axisymmetric Stoneley waves in a tungsten-aluminum interface as the example illustrated in Fig. 1. Material 1 is tungsten and material 2 - aluminum, characterized by [24-27]

$$
\begin{aligned}
& \bar{\rho}=19250 \mathrm{~kg} / \mathrm{m}^{3}, \quad \rho=2700 \mathrm{~kg} / \mathrm{m}^{3}, \\
& \bar{\lambda}=199.4 \times 10^{9} \mathrm{~Pa}, \quad \lambda=55.28 \times 10^{9} \mathrm{~Pa}, \\
& \bar{\mu}=158.56 \times 10^{9} \mathrm{~Pa}, \quad \mu=25.94 \times 10^{9} \mathrm{~Pa}
\end{aligned}
$$

which gives

$$
\begin{aligned}
& \bar{c}_{L}=5180 \mathrm{~m} / \mathrm{s}, \quad c_{L}=6300 \mathrm{~m} / \mathrm{s}, \\
& \bar{c}_{T}=2870 \mathrm{~m} / \mathrm{s}, \quad c_{T}=3100 \mathrm{~m} / \mathrm{s}, \\
& m=1.08, \quad \bar{g}=6.1126 .
\end{aligned}
$$

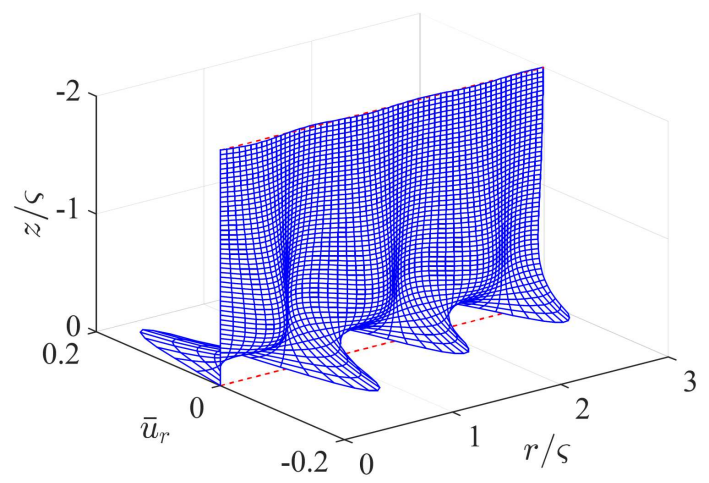

Fig. 2. Displacement $\bar{u}_{r}$ vs. the normalized coordinate $r / \varsigma$ and $z / \varsigma$ at $t=0$.

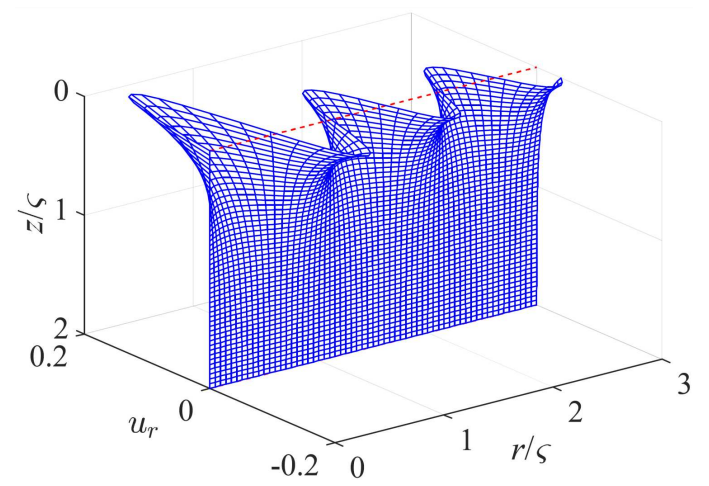

Fig. 3. As in Fig. 2 but for the displacement $u_{r}$.

The phase velocity is calculated from (37) as $c=2773.2171 \mathrm{~m} / \mathrm{s}$. Consequently, four parameters are

$$
\begin{array}{ll}
p=0.8979, & q=0.4467, \\
\bar{p} & =0.8446, \quad \bar{p}=0.2575,
\end{array}
$$

and three amplitude ratios are

$$
\bar{n}=2.5621, \quad n=0.2780, \quad h=0.4203
$$

with $k^{2} \bar{B}$ as the factor of the amplitudes. Since $k \varsigma=2 \pi$, the normalized displacements at $t=0$ become

$$
\begin{aligned}
\frac{\bar{u}_{r}}{k^{2} \bar{B}} & =\left[\bar{n} \mathrm{e}^{2 \pi \bar{p} z / \lambda}-\bar{q} \mathrm{e}^{2 \pi \bar{q} z / \lambda}\right] J_{1}\left(\frac{2 \pi r}{\lambda}\right), \\
\frac{u_{r}}{k^{2} \bar{B}} & =h\left[n \mathrm{e}^{2 \pi p z / \lambda}+q \mathrm{e}^{-2 \pi q z / \lambda}\right] J_{1}\left(\frac{2 \pi r}{\lambda}\right), \\
\frac{\bar{u}_{z}}{k^{2} \bar{B}} & =\left[-\bar{n} \bar{p} \mathrm{e}^{2 \pi p \bar{p} z / \lambda}+\mathrm{e}^{-2 \pi \bar{q} z / \lambda}\right] J_{0}\left(\frac{2 \pi r}{\lambda}\right), \\
\frac{u_{z}}{k^{2} \bar{B}} & =h\left[n p \mathrm{e}^{-2 \pi p z / \lambda}+\mathrm{e}^{-2 \pi q z / \lambda}\right] J_{0}\left(\frac{2 \pi r}{\lambda}\right) .
\end{aligned}
$$

The displacements at $t=0$ for Stoneley waves are plotted in Figs. 2-5, for the given structure in Fig. 1.

For a different time, normalized displacements at $z=0$ are plotted in Figs. 6 and 7 . 


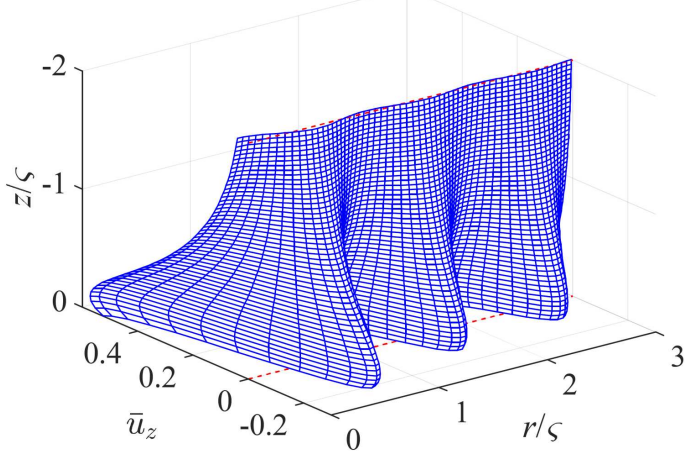

Fig. 4. Displacement $\bar{u}_{z}$ vs. the normalized coordinate $r / \varsigma$ and $z / \varsigma$ at $t=0$.

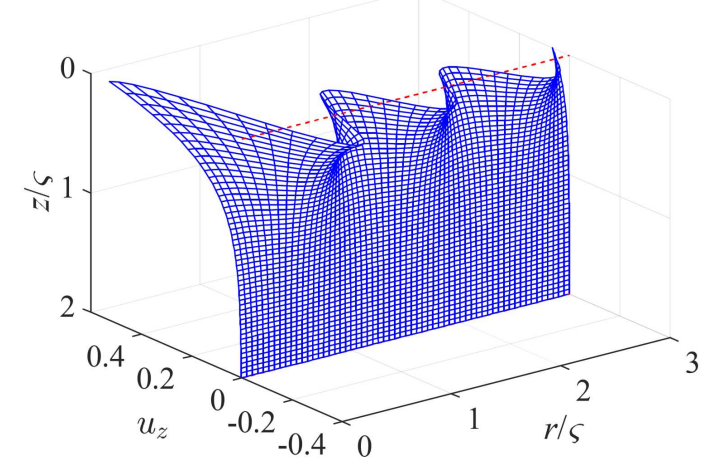

Fig. 5. As in Fig. 4 but for the displacement $u_{z}$.

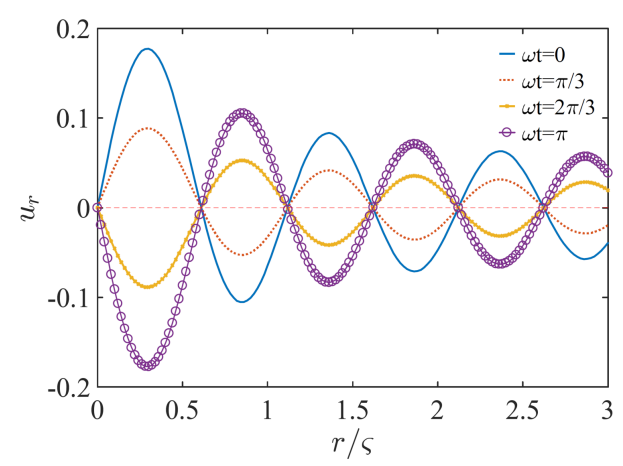

Fig. 6. Displacement $u_{r}$ (or $\bar{u}_{r}$ ) vs. $z / \varsigma$ at $z=0$.

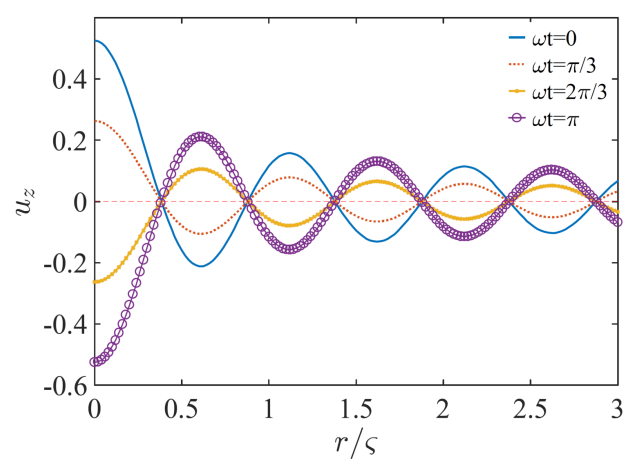

Fig. 7. As in Fig. 6 but for the displacement $u_{z}$ (or $\bar{u}_{z}$ ).

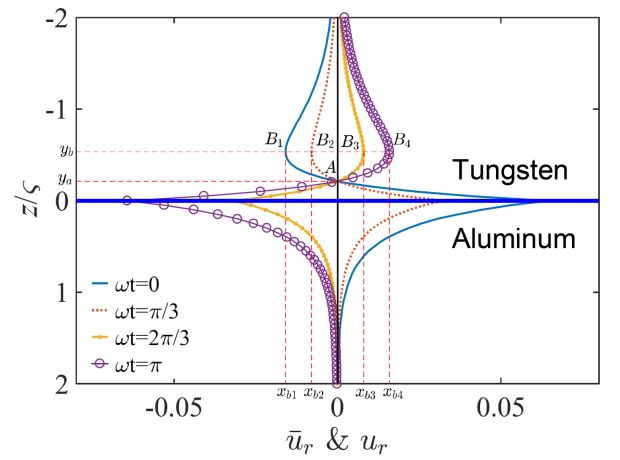

Fig. 8. Displacements $\bar{u}_{r}$ and $u_{r}$ at $r / \varsigma=1.25$ in the depth direction.

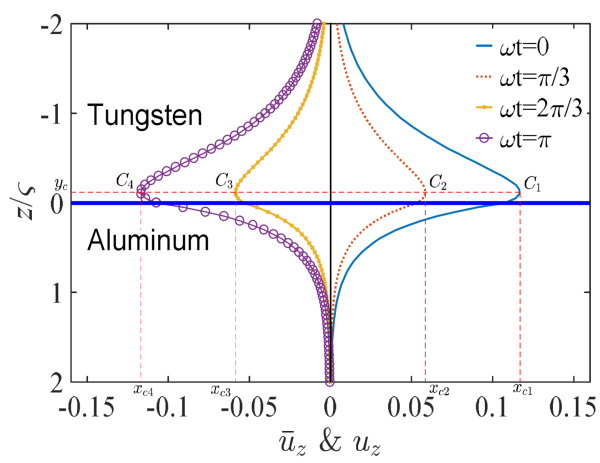

Fig. 9. As in Fig. 8 but for the displacements $\bar{u}_{z}$ and $u_{z}$.

With $r=1.25 \varsigma$, the displacements $\bar{u}_{r}$ and $u_{r}$ varied in time with the depth, as depicted in Fig. 8. Point $A$ is the site of the node for which the value on the vertical ordinate is $y_{a}$. Points $B_{1}, B_{2}, B_{3}$ and $B_{4}$ are the maximum displacements in the tungsten layer, respectively, for which values on the vertical ordinate are the same, i.e., $y_{b}$, while on the horizontal ordinate they are $x_{b 1}, x_{b 2}, x_{b 3}$ and $x_{b 4}$. Here $y_{a}=-0.2116, y_{b}=-0.5336$ and $x_{b 1}=2 x_{b 2}=-2 x_{b 3}=-x_{b 4}=-0.0159$.

Similarly, the displacements $\bar{u}_{z}$ and $u_{z}$ are shown in Fig. 9. There is no node and point $C$ is still the position of the maximum displacement, $y_{c}=-0.1201$ and $x_{c 1}=2 x_{c 2}=-2 x_{c 3}=$ $-x_{c 4}=0.1169$.

Finally, displacement solutions (41) can be used to study the axisymmetric Stoneley waves in the far field. Strictly speaking, the wave velocity is defined only by the function $f(k(r-c t))$. It can be clearly seen at this stage that displacements $u_{r}, u_{z}$ and $\bar{u}_{r}$, $\bar{u}_{z}$ do not satisfy this requirement. However, it is known that the Bessel functions can be expressed in the following asymptotic form:

$$
\begin{aligned}
& J_{1}(x)=\sqrt{\frac{2}{\pi x}} \sin (x-\pi / 4), \\
& J_{0}(x)=\sqrt{\frac{2}{\pi x}} \cos (x-\pi / 4),
\end{aligned}
$$

with such an approximation and for the large radius $r,(41)$ can be written as 


$$
\begin{aligned}
& u_{r}=a(z) \sin (k r-\pi / 4) \cos (\omega t), \\
& \bar{u}_{r}=\bar{a}(z) \sin (k r-\pi / 4) \cos (\omega t), \\
& u_{z}=b(z) \cos (k r-\pi / 4) \cos (\omega t), \\
& \bar{u}_{z}=\bar{b}(z) \cos (k r-\pi / 4) \cos (\omega t),
\end{aligned}
$$

where

$$
\begin{aligned}
& a(z)=h k^{2} \bar{B} \sqrt{\frac{2}{k \pi r}}\left(n \mathrm{e}^{-\alpha z}+q \mathrm{e}^{-\beta z}\right), \\
& \bar{a}(z)=k^{2} \bar{B} \sqrt{\frac{2}{k \pi r}}\left(\bar{n} \mathrm{e}^{\bar{\alpha} z}-\bar{q} \mathrm{e}^{\bar{\beta} z}\right), \\
& b(z)=h k^{2} \bar{B} \sqrt{\frac{2}{k \pi r}}\left(n p \mathrm{e}^{-\alpha z}+\mathrm{e}^{-\beta z}\right), \\
& \bar{b}(z)=k^{2} \bar{B} \sqrt{\frac{2}{k \pi r}}\left(-\bar{n} \bar{p} \mathrm{e}^{\bar{\alpha} z}+\mathrm{e}^{\bar{\beta} z}\right) .
\end{aligned}
$$

Using the basic identities of trigonometric functions, one can even write

$$
\begin{aligned}
& u_{r}=a(z) \frac{\sin \left(k r-\frac{\pi}{4}+\omega t\right)+\sin \left(k r-\frac{\pi}{4}-\omega t\right)}{2}, \\
& \bar{u}_{r}=\bar{a}(z) \frac{\sin \left(k r-\frac{\pi}{4}+\omega t\right)+\sin \left(k r-\frac{\pi}{4}-\omega t\right)}{2}, \\
& u_{z}=b(z) \frac{\cos \left(k r-\frac{\pi}{4}+\omega t\right)+\cos \left(k r-\frac{\pi}{4}-\omega t\right)}{2}, \\
& \bar{u}_{z}=\bar{b}(z) \frac{\cos \left(k r-\frac{\pi}{4}+\omega t\right)+\cos \left(k r-\frac{\pi}{4}-\omega t\right)}{2} .
\end{aligned}
$$

The above results are in the waveform of the $f(k(r-c t))$ pattern and are the superposition of one forward travelling wave with the one backward travelling wave with the same wave velocity $c=\omega / k$. The waves thus exhibit the property of travelling waves. Furthermore, the displacements in the far field exhibit a $r^{-1 / 2}$ decaying, and it is invariant in Cartesian coordinates. In fact, it can be explained by the density of the energy in the propagation. When the axisymmetric Stoneley waves travel from the source, the energy is distributed on the cylindrical surface where each infinitesimal area possesses the energy proportional to $1 /(2 \pi r)$.

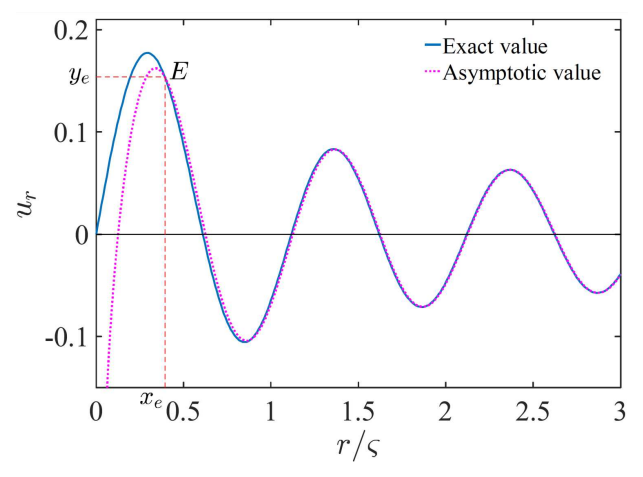

Fig. 10. A comparison of $u_{r}$ (or $\bar{u}_{r}$ ) with exact and asymptotic values at $z=0$ and $t=0$.
TABLE I

A comparison of $u_{r}$ (or $\bar{u}_{r}$ ) with exact and asymptotic values at $z=0$ and $t=0$.

\begin{tabular}{c|c|c|c}
\hline \hline$r / \varsigma$ & $\begin{array}{c}\text { Exact } \\
\text { value }\left[\times 10^{-2}\right]\end{array}$ & $\begin{array}{c}\text { Asymptotic } \\
\text { value }\left[\times 10^{-2}\right]\end{array}$ & $\begin{array}{c}\text { error } \\
{[\%]}\end{array}$ \\
\hline 0.5 & 8.67 & 9.70 & 11.84 \\
1 & -6.47 & -6.86 & 5.98 \\
1.5 & 5.38 & 5.60 & 3.99 \\
2 & -4.71 & -4.85 & 2.99 \\
2.5 & 4.23 & 4.34 & 2.39 \\
3 & -3.88 & -3.96 & 1.99
\end{tabular}

TABLE II

A comparison of $u_{z}\left(\right.$ or $\left.\bar{u}_{z}\right)$ with exact and asymptotic values at $z=0$ and $t=0$.

\begin{tabular}{c|c|c|c}
\hline \hline$r / \varsigma$ & $\begin{array}{c}\text { Exact } \\
\text { value }\left[\times 10^{-2}\right]\end{array}$ & $\begin{array}{c}\text { Asymptotic } \\
\text { value }\left[\times 10^{-2}\right]\end{array}$ & $\begin{array}{c}\text { error } \\
{[\%]}\end{array}$ \\
\hline 0.5 & -15.98 & -16.72 & 4.62 \\
1 & 11.57 & 11.82 & 2.18 \\
1.5 & -9.52 & -9.65 & 1.42 \\
2 & 8.27 & 8.36 & 1.05 \\
2.5 & -7.42 & -7.48 & 0.83 \\
3 & 6.78 & 6.83 & 0.69
\end{tabular}

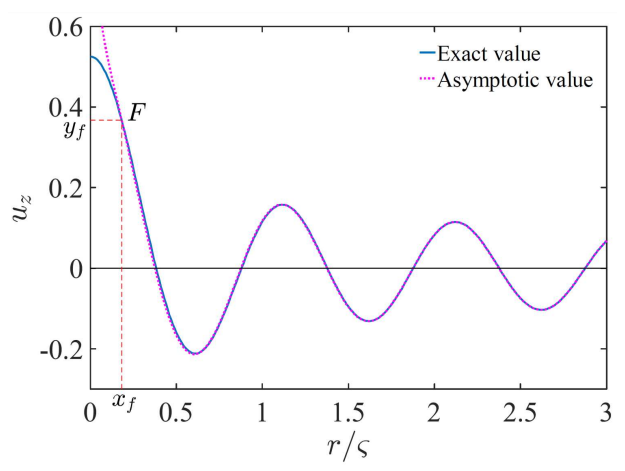

Fig. 11. As in Fig. 10 but for the comparison of $u_{z}\left(\right.$ or $\left.\bar{u}_{z}\right)$.

Because displacements are proportional to the square root of energy, the displacements are proportional to $r^{-1 / 2}$. For a large radius, the axisymmetric Stoneley waves can be considered as planar surface waves in a small range. With $z=0$, $u_{r}$ or $\bar{u}_{r}$ using plots in Fig. 10, where point $E$ is the first intersection between the exact and approximate curves, having $x_{e}=0.3930$ and $y_{e}=0.1537$ and numerical comparisons in Table I, the accuracy of Cartesian solutions near the source is clearly shown. Also, $u_{z}$ or $\bar{u}_{z}$ using plots in Fig. 11, where point $\mathrm{F}$ is still the first intersection, having $x_{f}=$ 0.1819 and $y_{f}=0.3673$ and numerical comparisons in Table II. 
Our results show that the plane Stoneley waves can be used to approximate the far-field axisymmetric surface waves. In addition, this also explains that the displacement near the origin or the excitation point is larger than that of the outer region which can be well represented in Cartesian coordinates. This also interprets that the damage of the epicentre earthquake is much more serious than that of the distant area, even the displacement is not reduced by the distance if damping is not considered. Of course, with steady waves generated by point sources, axisymmetric coordinates and solutions must be used to better understand wave propagation, especially near the source or near field. This is also true that acoustic wave devices or possible axisymmetric structures under point source excitation would enhance the wave characteristics required in the application at the centre of the circular structure.

\section{Conclusions}

With systematically deduced solutions which exist as Stoneley waves of the structure of two perfectly bounded half-spaces in cylindrical coordinates the conclusion can be drawn that the wave velocity still maintains the same value as in Cartesian coordinates. Expectedly, due to the nature of the Bessel function, the similar displacements in the two half-spaces are attenuated to the constant value as the known results in Cartesian coordinates. In other words, the particle polarization trajectory of the Stoneley wave near the origin is slightly different from Cartesian coordinates but the asymptotic expansion of the Bessel function shows that the displacement amplitude will remain the same as Cartesian coordinates when the radius is large or far from the origin. At the same time, the results further show that the axisymmetric solution near the center is larger than the solution given by the trigonometric function which implies that the axisymmetric description is more accurate in solving such problems. In the case of a wavelength with the dimension of kilometers, a better solution can be obtained by using the cylindrical coordinate expression proposed in recent studies. In addition, these results of the Bessel functions would also be offered as the basis for the study of sensors and actuators in finite structures related to Stoneley waves. Stoneley waves tested and measured from a point source also require a better understanding on the displacement changed near and far from the origin. The solutions and analysis in this regard are undoubtedly a good start for studying Stoneley waves in curved structures; circular devices with a systematic optimization should particularly be considered.

\section{Acknowledgments}

This research was supported in part by the National Natural Science Foundation of China (grants 11672142 and 11372145) and the K.C. Wong
Magana Fund of Ningbo University. Additional support was granted through the Technology Innovation 2025 Program (grant 2019B10122) of the Municipality of Ningbo.

\section{References}

[1] W.M. Ewing, W.S. Jardetzky, F. Press, Elastic Waves in Layered Media, McGrawHill, 1957.

[2] J.D. Achenbach, Wave Propagation in Elastic Solids, North-Holland, 1973.

[3] K.F. Graff, Wave Motion in Elastic Solids, Dover, 1975.

[4] A.C. Eringen, Elastodynamics, Vol. 2, Linear Theory, Academic Press, 1975.

[5] J.L. Rose, Ultrasonic Guided Waves in Solid Media, Cambridge University Press, 2014.

[6] J. Wang, J.B. Lin, J. Intel. Mat. Syst. Str. 16, 623 (2005).

[7] J. Wang, J.B. Lin, Y.P. Wan, Z. Zhong, Int. J. Appl. Electromagn. 22, 53 (2005).

[8] J. Wang, K.Y. Hashimoto, J. Sound Vibrat. 295, 838 (2006).

[9] J. Wang, J.K. Du, Q.Q. Pan, Sci. China Phys. Mech. 10, 631 (2007).

[10] R. Stoneley, Proc. R. Soc. Lond. A 106, 416 (1924).

[11] J. Wang, S.Y. Wang, L.T. Xie, Y.Y. Zhang, L.L. Yuan, J.K. Du, H. Zhang, Theor. Appl. Mech. Lett. 10, 120 (2020).

[12] K. Sezawa, K. Kanai, Bull. Earthquake Res. Inst. Tokyo Univ. 17, 1 (1939).

[13] J.G. Scholte, Proc. Konink. Nederl. Akad. Weten Schappen 45, 159 (1942).

[14] J.G. Scholte, Geophys. J. In. 5, 120 (1947).

[15] L. Cagniard, Reflection and Refraction of Progressive Seismic Waves, McGraw-Hill, 1962.

[16] M. Pasternak, Acta Phys. Pol. A 114, 169 (2008).

[17] D. Mitchell, J.T. Gau, Eur. J. Mech. A Solid 75, 216 (2019).

[18] P. Sofer, M. Sofer, J. Gebauer, P. Niegodajew, K. Gruszka, Acta Phys. Pol. A 138, 272 (2020).

[19] M. Pasternak, Acta Phys. Pol. A 116, 371 (2009).

[20] R.M.A. Stoneley, Geophys. Suppl. Monthly Notices R. Astron. Soc. 1, 527 (1928).

[21] A.S. Ginzbarg, E. Strick, Bull. Seismol. Soc. Am. 48, 51 (1958). 
[22] D. Royer, E. Dieulesaint, Elastic Waves in Solids I and II, Springer, 2000.

[23] J. Kaplunov, D. Prikazchikov, Adv. Appl. Mech. 50, 1 (2017).

[24] F.M. Esteban, C.M. Manuel, F.G. Norberto, S.M. Ricardo, R.C. Alejandro, J. Appl. Math. 313207, 1 (2012).

[25] J. Qian, C.P. Li, L.R. Qian, M.J. Li, H.J. Li, B.H. Yang, Diam. Relat. Mater. 88, 123 (2018).

[26] M.T. Chen, Q. Huan, F.X. Li, J. Acoust. Soc. Am. 146, 482 (2019).

[27] D.M. Giancarlo, Sci. Rep. (UK) 10, 211 (2020). 